\title{
Skitouren-Boom in den Alpen - Fluch oder Segen aus sportmedizinischer Sicht?
}

\author{
Stefan Fröhlich \\ Universitäres Zentrum für Prävention und Sportmedizin, Universitätsklinik Balgrist, Zürich
}

Skitouren erfreuen sich in den letzten Jahren einer stetig wachsenden Popularität [1]. In Deutschland beispielsweise hat sich die Anzahl der Skitourengeher/innen in den vergangenen zehn Jahren verdreifacht [2]. Von ähnlichen Zuwachsquoten ist auch in den anderen Alpenländern auszugehen. Doch wie ist diese Trendsportart aus medizinischer Sicht zu beurteilen? Dem pauschalen Urteil, dass «Skitourengehen gesund ist», würde sich sicherlich die überwiegende Mehrzahl der Expertinnen und Experten anschliessen. Den zahlreichen positiven Aspekten, die insbesondere das Ausdauertraining ebenso wie das Training von Kraft und koordinativen Fähigkeiten umfassen, stehen jedoch relevante, keinesfalls zu unterschätzende Gefahren gegenüber. Insbesondere fatale Lawinenunfälle führen immer wieder dazu, dass dieser Natursport traurige Schlagzeilen macht. Pro Jahr sterben in den Schweizer Alpen im langjährigen Mittel ca. 25 Personen durch Lawinenabgänge, die meisten davon sind Skitourengeher/innen [3]. Die präventiven Massnahmen zur Verminderung dieser Gefahr sind hinreichend bekannt und werden in der Fachwelt immer wieder thematisiert. Kaum Beachtung finden dagegen die zahlreichen nicht-tödlichen Unfälle der Skitourengeher/innen, da diese von der Öffentlichkeit relativ unbemerkt ablaufen. Diesem Problem widmet sich der Artikel «Stürze bei Skitouren - eine Analyse aus den Schweizer Alpen» [4]. Die Epidemiologie der Skitourenstürze wird aufgezeigt, und die relevanten Problembereiche werden erläutert. Wenn diese bekannt sind und ins Bewusstsein gerückt werden, können auch wirksame Präventionsmassnahmen entwickelt und etabliert werden. Denkbar sind auf der einen Seite präventive Trainingsmassnahmen - deren standardisierte Umsetzung sowie die Überprüfung ihrer Wirksamkeit stellen allerdings eine grosse Herausforderung dar. Auf der anderen Seite rückt das Material in den Fokus: So selbstverständlich wie die Lawinenausrüstung zur Vermeidung eines fatalen Lawinenunglücks ins Gepäck einer Skitourengeherin/eines Skitourengehers gehört, so sollte auch eine entsprechend gut funktionierende und zertifizierte Sicherheitsbindung mit auf Tour kommen. Der Trend geht bei der Ausrüstung allerdings immer mehr zur Gewichtsoptimierung, nicht selten zu Lasten der Sicherheit. Manche Modelle lösen zwar im Abfahrtsmodus, nicht jedoch im Aufstiegsmodus aus, obwohl technisch schwierige Aufstiegspassagen durchaus gefährliche Stürze provozieren können. Andere Modelle besitzen gar keine oder nur eine rudimentäre, nicht zertifizierte Auslösefunktion. Wenn hier bereits bei der Materialauswahl der Sicherheitsgedanke eine zentrale Rolle einnimmt und nicht nur die Gewichtsminimierung, könnten die Verletzungszahlen, insbesondere an den Knien, mit grosser Wahrscheinlichkeit reduziert werden. Und mit niedrigeren Verletzungsraten würden die zahlreichen zweifellos vorhandenen - gesundheitswirksamen Aspekte dieser Sportart noch weiter in den Vordergrund rücken.

\section{Bibliografie}

1. Mueller T, Ruedl G, Ernstbrunner M, et al. A prospective injury surveillance study on ski touring. Orthop J Sports Med. 2019;7:2325967119867676.

2. Heubacher A. Skitouren-Boom: Warnung vor Chaos auf Tirols Bergen. Tiroler Tageszeitung 2020. https://www.tt.com/ artikel/16515866/skitouren-boom-warnung-vor-chaosauf-tirols-bergen; letzter Zugriff: 02.04.2020.

3. WSL-Institut für Schnee- und Lawinenforschung SLF 2019. https://www.slf.ch/de/lawinen/unfaelle-und-schadenlawinen/ langjaehrige-statistiken.html; letzter Zugriff: 02.04.2020.

4. Gasser B. Sturzverletzungen bei Skitouren - eine retrospektive Analyse aus den Schweizer Alpen. Praxis. 2020;109:11211126.

Dr. med.Stefan Fröhlich, Dipl.-Sportwiss. Univ.

Universitätsklinik Balgrist

Universitäres Zentrum für Prävention

und Sportmedizin

Forchstrasse 319

8008 Zürich

stefan.froehlich@balgrist.ch 